\title{
The (pro)renin receptor: what's in a name?
}

\section{Matias Simons, Michael Bader and Dominik N. Müller $\mathbb{D}$}

We read with interest the recent Review by A. Ichihara and M. S. Yatabe (The (pro)renin receptor in health and disease. Nat. Rev. Nephrol. 15, 693-712 (2019)) $)^{1}$. The (pro)renin receptor (PRR; also known as ATP6AP2) was first discovered in 1998 in a biochemical purification approach that yielded a $8.9 \mathrm{kDa}$ fragment of a membrane-associated protein. This fragment associated with the proton pump V-ATPase and was hence given the gene name ATP6AP2 (REF.). However, in 2002, a seminal paper by Nguyen and colleagues ${ }^{3}$ suggested that the fragment belonged to a larger protein - the PRR - that can bind to prorenin, the precursor of renin, on the cell surface, thereby facilitating its processing to renin. The circulatory levels of renin are normally ten times lower than those of prorenin. Moreover, elevated prorenin levels correlate with the microvascular complications of diabetes and hypertension, suggesting that prorenin may contribute to the extra-renal effects of renin. The PRR therefore represented a promising drug target to prevent end-organ damage mediated by the reninangiotensin system (RAS). As a result, the PRR attracted a lot of attention, which was aided to a large extent by its flashy name.

Despite almost two decades of PRR research, it is still far from clear how prorenin is processed and, most importantly, whether this processing is physiologically relevant for the RAS. Unlike previous reviews of this research $\operatorname{area}^{4,5}$, the article by Ichihara and Yatabe and another recent review by N. Ramkumar and D. E. Kohan published in Kidney International ${ }^{6}$ do not acknowledge this uncertainty.

A major issue plaguing the field is that deletion of the ATP6AP2 gene causes embryonic lethality. For this reason, most loss-of-function studies either use a blocking peptide against the handle region or conditional mouse knockouts. Whereas the efficiency of the peptide approach remains controversial $^{7}$, the conditional knockout approach has so far mainly revealed V-ATPasedependent effects, as reflected by reduced lysosomal acidification and/or impaired cell homeostasis in the targeted cell types and tissues. Importantly, impairment of cell homeostasis and even slow death of PRR-deficient cells can easily be missed if researchers do not specifically look for these effects ${ }^{8,9}$. Therefore, it is likely that putative RAS-related phenotypes reported in mice ${ }^{10,11}$ are influenced or even caused by impaired cell homeostasis.

Studies of the effects of mutations in the ATP6AP2 gene in humans have failed to identify any evidence of a role of the encoded protein in the RAS ${ }^{12,13}$. Instead, one report provided evidence for a causal relationship of ATP6AP2 missense mutations with steatohepatitis, immunodeficiency and psychomotor impairment ${ }^{14}$. The most prominent cellular phenotypes were lysosomal and/or autophagic defects. The similarity of these phenotypes with phenotypes that are associated with deficiencies of endoplasmic reticulum (ER)-resident V-ATPase assembly factors as well as cell biological studies focusing on an ER retention motif in the cytosolic tail led to the proposal that the PRR could be a true component of the assembly machinery ${ }^{14,15}$. Defects in V-ATPase assembly may explain decreased endolysosomal acidification in the setting of human ATP6AP2 mutations as well as Wnt signalling phenotypes reported for model organisms with reduced ATP6AP2 $\left(\right.$ REFS $\left.^{16,17}\right)$. However, the ER localization of $\mathrm{V}$-ATPase assembly is difficult to reconcile with any prorenin-binding activity for the PRR at the cell surface.

William Shakespeare's Juliet famously asks "what's in a name?". In the case of the PRR, the name has had a profound impact on the development of an entire research field. Although the two recent reviews clearly point out that the PRR has pleiotropic functions, noncritical use of this name could be misleading for the research community.

Matias Simons ${ }^{1,2}{ }^{凶}$, Michael Bader $3,4,5,6$ and Dominik N. Müller (iD ${ }^{3,6,7}$

'INSERM UMR 1163, Laboratory of Epithelial Biology and Disease, Imagine Institute, Paris Descartes University,
Sorbonne Paris Cité, Hôpital Necker-Enfants Malades,

Paris, France.

2 Institute of Human Genetics, University Hospital Heidelberg, Heidelberg, Germany. ${ }^{3}$ Max-Delbrück Center for Molecular Medicine (MDC), Berlin-Buch, Germany. ${ }^{4}$ University of Lübeck, Institute for Biology, Lübeck, Germany.

${ }^{5}$ Charité-Universitätsmedizin Berlin, Berlin, Germany. ${ }^{6}$ German Center for Cardiovascular Research (DZHK), Partner Site Berlin, Berlin, Germany. Experimental and Clinical Research Center, a joint cooperation of Max-Delbrück Center for Molecular Medicine and Charité-Universitätsmedizin Berlin, Berlin, Germany.

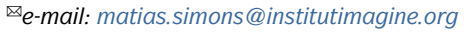
https://doi.org/10.1038/s41581-020-0274-9

Ichihara, A. \& Yatabe, M. S. The (pro)renin receptor in health and disease. Nat. Rev. Nephrol. 15, 693-712 (2019).

2. Ludwig, J. et al. Identification and characterization of a novel 9.2-kDa membrane sector-associated protein of vacuolar proton-ATPase from chromaffin granules. J. Biol. Chem. 273, 10939-10947 (1998).

3. Nguyen, G. et al. Pivotal role of the renin/prorenin receptor in angiotensin II production and cellular responses to renin. J. Clin. Invest. 109, 1417-1427 (2002).

4. Sihn, G., Rousselle, A., Vilianovitch, L., Burckle, C. $\&$ Bader, M. Physiology of the (pro)renin receptor: Wnt of change? Kidney Int. 78, 246-256 (2010).

5. Danser, A. H. The role of the (pro)renin receptor in hypertensive disease. Am. J. Hypertens. 28, 1187-1196 (2015).

6. Ramkumar, N. \& Kohan, D. E. The (pro)renin receptor an emerging player in hypertension and metabolic syndrome. Kidney Int. 95, 1041-1052 (2019).

7. Binger, K. J. \& Muller, D. N. Autophagy and the (pro) renin receptor. Front. Endocrinol. 4, 155 (2013).

8. Ramkumar, N. et al. Collecting duct principal, but not intercalated, cell prorenin receptor regulates renal sodium and water excretion. Am. J. Physiol. Renal Physiol. 315, F607-F617 (2018).

9. Trepiccione, F. et al. Renal Atp6ap2/(pro)renin receptor is required for normal vacuolar $\mathrm{H}^{+}$-ATPase function but not for the renin-angiotensin system. J. Am. Soc. Nephrol. 27, 3320-3330 (2016).

10. Li, W. et al. Neuron-specific (pro)renin receptor knockout prevents the development of salt-sensitive hypertension. Hypertension 63, 316-323 (2014).

11. Peng, K. et al. Collecting duct (pro)renin receptor targets $\mathrm{ENaC}$ to mediate angiotensin II-induced hypertension. Am. J. Physiol. Renal Physiol. 312 F245-F253 (2017).

12. Hirose, T. et al. ATP6AP2 variant impairs CNS development and neuronal survival to cause fulminant neurodegeneration. J. Clin. Invest. 129, 2145-2162 (2019).

13. Korvatska, O. et al. Altered splicing of ATP6AP2 causes X-linked parkinsonism with spasticity (XPDS). Hum. Mol. Genet. 22, 3259-3268 (2013).

14. Rujano, M. A. et al. Mutations in the X-linked ATP6AP2 cause a glycosylation disorder with autophagic defects. J. Exp. Med. 214, 3707-3729 (2017).

15. Guida, M. C. et al. ATP6AP2 functions as a V-ATPase assembly factor in the endoplasmic reticulum. Mol. Biol. Cell 29, 2156-2164 (2018).

16. Cruciat, C. M. et al. Requirement of prorenin receptor and vacuolar $\mathrm{H}^{+}$-ATPase-mediated acidification for Wnt signaling. Science 327, 459-463 (2010)

17. Hermle, T., Saltukoglu, D., Grunewald, J., Walz, G. \& Simons, M. Regulation of Frizzled-dependent planar polarity signaling by a V-ATPase subunit. Curr. Biol. 20, 1269-1276 (2010).

Competing interests

The authors declare no competing interests. 